Physics

Physics Research Publications

\title{
Present status of controversies regarding the thermal Casimir force
}
V. M. Mostepanenko*
V. B. Bezerra ${ }^{\dagger}$
R. S. Decca ${ }^{\ddagger}$
B. Geyer**
E. Fischbach ${ }^{\dagger \dagger}$
L. Klimchitskaya ${ }^{\ddagger \ddagger}$
D. E. Krause ${ }^{\S}$
D. Lopez
C. Romer"

$*$
$\dagger$
$\ddagger$
$* *$
$\dagger \dagger$
$\ddagger \ddagger$
$\S$
$\uparrow$
$\|$

This paper is posted at Purdue e-Pubs.

http://docs.lib.purdue.edu/physics_articles/326 


\title{
Present status of controversies regarding the thermal Casimir force
}

\author{
V M Mostepanenko 1 , V B Bezerra ${ }^{2}$, R S Decca ${ }^{3}$, B Geyer ${ }^{4}$, E Fischbach $^{5}$, \\ G L Klimchitskaya ${ }^{6}$, D E Krause ${ }^{7,5}$, D López ${ }^{8}$ and C Romero ${ }^{2}$
}

${ }^{1}$ Noncommercial Partnership ‘Scientific Instruments', Moscow, Russia

2 Department of Physics, Federal University of Paraiba, João Pessoa, Brazil

${ }^{3}$ Department of Physics, Indiana University-Purdue University Indianapolis, Indianapolis, IN 46202, USA

${ }^{4}$ Center of Theoretical Studies and Institute for Theoretical Physics, Leipzig University, Augustusplatz 10/11, D-04109 Leipzig, Germany

${ }^{5}$ Department of Physics, Purdue University, West Lafayette, IN 47907, USA

${ }^{6}$ North-West Technical University, Millionnaya Street 5, St Petersburg, Russia

7 Department of Physics, Wabash College, Crawfordsville, IN 47933, USA

${ }^{8}$ Bell Laboratories, Lucent Technologies, Murray Hill, NJ 07974, USA

Received 25 October 2005, in final form 15 December 2005

Published 10 May 2006

Online at stacks.iop.org/JPhysA/39/6589

\begin{abstract}
It is well known that, beginning in 2000, the behaviour of the thermal correction to the Casimir force between real metals has been hotly debated. As was shown by several research groups, the Lifshitz theory, which provides the theoretical foundation for the calculation of both the van der Waals and Casimir forces, leads to different results depending on the model of metal conductivity used. To resolve these controversies, theoretical considerations based on the principles of thermodynamics and new experimental tests were invoked. We analyse the present status of the problem (in particular, the advantages and disadvantages of the approaches based on the surface impedance and on the Drude model dielectric function) using rigorous analytical calculations of the entropy of a fluctuating field. We also discuss the results of a new precise experiment on the determination of the Casimir pressure between two parallel plates by means of a micromechanical torsional oscillator.
\end{abstract}

PACS numbers: $12.20 . \mathrm{Fv}, 12.20 . \mathrm{Ds}, 11.10 . \mathrm{Wx}, 05.70 .-\mathrm{a}$

\section{Introduction: Lifshitz formula provides the theoretical background for the thermal Casimir force}

Since the beginning of 2000, the dependence of the Casimir force acting between real metals on separation and temperature has been hotly debated. It is well known that van der Waals and Casimir forces act between closely spaced macroscopic bodies. Both forces are of the same 
physical nature and are caused by long wavelength electromagnetic fluctuations [1]. The name 'van der Waals' is usually used in a nonrelativistic case when the force and other physical quantities depend on the Planck constant $\hbar$, but do not depend on the velocity of light $c$. In the relativistic case, which corresponds to larger separations, the name 'Casimir' is commonly used, since he was the first to predict the force acting in vacuum between two parallel uncharged plates made of ideal metals [2]. During the last few years, dispersion forces (which is the generic name for both kinds of forces caused by fluctuations) were actively investigated, not only theoretically but also experimentally (see the monographs [3-5] and a review [6]). This was motivated by many prospective applications of dispersion phenomena in fundamental physics and nanotechnology. In particular, the problem of an accurate theoretical description of dispersion forces between real bodies (i.e. taking into account the realistic conductivity properties, surface roughness and nonzero temperature) took on great significance. This paper is devoted to the outstanding problems arising in the case of plates made of real metals and having nonzero temperature.

The free energy of the dispersion interaction between two thick parallel plates (semispaces) described by a dielectric permittivity $\varepsilon(\omega)$, with a gap of width $z$ between them in thermal equilibrium at temperature $T$, was derived by Lifshitz [7]. It can be represented in the form

$$
\begin{aligned}
\mathcal{F}(z, T)=\frac{k_{\mathrm{B}} T}{2 \pi} & \sum_{l=0}^{\infty}\left(1-\frac{1}{2} \delta_{l 0}\right) \int_{0}^{\infty} k_{\perp} \mathrm{d} k_{\perp} \\
& \times\left\{\ln \left[1-r_{\|}^{2}\left(\xi_{l}, k_{\perp}\right) \mathrm{e}^{-2 q_{l} z}\right]+\ln \left[1-r_{\perp}^{2}\left(\xi_{l}, k_{\perp}\right) \mathrm{e}^{-2 q_{l} z}\right]\right\} .
\end{aligned}
$$

Here $k_{\mathrm{B}}$ is the Boltzmann constant, $\xi_{l}=2 \pi k_{\mathrm{B}} T l / \hbar$ are the Matsubara frequencies, $q_{l}=$ $\left(k_{\perp}^{2}+\xi_{l}^{2} / c^{2}\right)^{1 / 2}, k_{\perp}$ is the projection of the wave vector in the plane of the plates and $r_{\|, \perp}\left(\xi_{l}, k_{\perp}\right)$ are the reflection coefficients for two independent polarizations of the electromagnetic field. The explicit form of these coefficients in the case of real metals is closely connected to the controversies which are the subject of this paper.

\section{Two main approaches to the presentation of reflection coefficients: the dielectric permittivity and the surface impedance}

In the framework of the first approach, the reflection coefficients are expressed in terms of the dielectric permittivity of a plate material. In application to equation (1) this approach goes back to Lifshitz who used it to describe the Casimir and van der Waals forces between dielectrics. In this case

$$
r_{\|} \equiv r_{\|}^{L}\left(\xi_{l}, k_{\perp}\right)=\frac{\varepsilon_{l} q_{l}-k_{l}}{\varepsilon_{l} q_{l}+k_{l}}, \quad r_{\perp} \equiv r_{\perp}^{L}\left(\xi_{l}, k_{\perp}\right)=\frac{k_{l}-q_{l}}{k_{l}+q_{l}},
$$

where $k_{l}=\left(k_{\perp}^{2}+\varepsilon_{l} \xi_{l}^{2} / c^{2}\right)^{1 / 2}$ and $\varepsilon_{l} \equiv \varepsilon\left(\mathrm{i} \xi_{l}\right)$. Within the Lifshitz approach, the fluctuating electromagnetic field is considered both in the gap and in the interior of the dielectric semispaces with the continuity boundary conditions on their surfaces

$$
E_{1 t}=E_{2 t}, \quad B_{1 t}=B_{2 t}, \quad D_{1 n}=D_{2 n}, \quad B_{1 n}=B_{2 n}
$$

(note that we do not consider ferromagnets and set $\boldsymbol{B}=\boldsymbol{H}$ ).

Of prime importance is the contribution of the free energy in equation (1) with $l=0$. It is well known that this term determines the total value of $\mathcal{F}$ at high temperatures (large separations) because all contributions with $l \geqslant 1$ are exponentially small in this limiting case $[5,6]$. Equation (1) with reflection coefficients (2) correctly describes the case of plates made of ideal metals if one uses the so-called Schwinger prescription [5, 6], i.e., take limit $\varepsilon \rightarrow \infty$ 
first and set $l=0$ afterwards. In doing so for ideal metals the equations

$$
r_{\|}^{L}\left(0, k_{\perp}\right)=r_{\perp}^{L}\left(0, k_{\perp}\right)=1
$$

are obtained which coincide with those obtained from thermal quantum field theory with Dirichlet boundary conditions [8] independent of the Lifshitz formula.

In [9] an attempt was made to describe the dispersion interaction between real metals by the Lifshitz formula using the Drude model to characterize their dielectric properties at low frequencies:

$$
\varepsilon(\mathrm{i} \xi)=1+\frac{\omega_{\mathrm{p}}^{2}}{\xi[\xi+\gamma(T)]} \approx 1+\frac{\omega_{\mathrm{p}}^{2}}{\xi \gamma(T)},
$$

where $\omega_{\mathrm{p}}$ is the plasma frequency and $\gamma(T)$ is the relaxation parameter. Substituting equation (5) into equation (2) one obtains

$$
r_{\|}^{L}\left(0, k_{\perp}\right)=1, \quad r_{\perp}^{L}\left(0, k_{\perp}\right)=0 .
$$

Equation (6) is preserved also in the limit of ideal metals, and is thus in contradiction with equation (4). If, however, one characterizes a metal by the plasma model,

$$
\varepsilon(\mathrm{i} \xi)=1+\frac{\omega_{\mathrm{p}}^{2}}{\xi^{2}},
$$

and extrapolates it to low frequencies, the reflection coefficients become

$$
r_{\|}^{L}\left(0, k_{\perp}\right)=1, \quad r_{\perp}^{L}\left(0, k_{\perp}\right)=\frac{\sqrt{c^{2} k_{\perp}^{2}+\omega_{\mathrm{p}}^{2}}-c k_{\perp}}{\sqrt{c^{2} k_{\perp}^{2}+\omega_{\mathrm{p}}^{2}}+c k_{\perp}} .
$$

Equation (8) agrees with the case of ideal metals (4) because $r_{\perp}^{L}\left(0, k_{\perp}\right) \rightarrow 1$ when $\omega_{\mathrm{p}} \rightarrow \infty$.

The approach to the theoretical description of the thermal Casimir force between real metals based on equation (6) was supported and developed in [10-13], while that following equations (7) and (8) was proposed in $[14,15]$. By convention we refer to them below as 'the Drude model approach' and 'the plasma model approach', respectively.

The Drude model approach predicts relatively large thermal corrections to the Casimir force between real metals at short separations of about 500 times greater than between ideal metals. At large separations or high temperatures the Drude model approach predicts $1 / 2$ the magnitude of the Casimir free energy and force than between ideal metals. (The same prediction was obtained using the model of a conducting wall by the classical Coulomb fluid [16] or 'nonrelativistic quantum electrodynamics' [17].) This prediction is in contradiction with the classical limit based on Kirchhoff's law [18]. As to the plasma model approach, its predictions are in qualitative agreement with the case of ideal metals. Because of this, the plasma model approach is called 'traditional' and the Drude model approach is called 'alternative'. The apparent advantage of the Drude model approach is that equation (5) presents the same behaviour of $\varepsilon \sim \omega^{-1}$ at low frequencies, as given by the Maxwell equations, whereas the behaviour $\varepsilon \sim \omega^{-2}$ in equation (7) is characteristic for the region of infrared optics, raising a question on the correctness of its extrapolation to low frequencies. On the other hand, equation (8) is in agreement with the limiting case of an ideal metal, whereas equation (6) is not. Bearing in mind that the description of real metals using $\varepsilon$ depending only on $\omega$ is not universal, it is worthwhile to use alternative physical quantities to characterize the reflection properties of metal surfaces.

In the framework of the second approach, which goes back to Ginzburg and his collaborators (see, e.g., [19]), the reflection coefficients in the Lifshitz formula are expressed in terms of the Leontovich impedance $Z(\omega)$. The Leontovich impedance boundary condition

$$
\boldsymbol{E}_{\mathrm{t}}=Z(\omega)\left[\boldsymbol{H}_{\mathrm{t}} \times \boldsymbol{n}\right]
$$


(where $\boldsymbol{n}$ is an internal unit vector directed into the medium and normal to the boundary) is approximate and permits one to find the electromagnetic field in the gap without considering it in the interior of the plates. In terms of the Leontovich impedance, the reflection coefficients in equation (1) are given by

$$
r_{\|} \equiv r_{\|}^{\mathrm{G}}\left(\xi_{l}, k_{\perp}\right)=\frac{c q_{l}-Z_{l} \xi_{l}}{c q_{l}+Z_{l} \xi_{l}}, \quad r_{\perp} \equiv r_{\perp}^{\mathrm{G}}\left(\xi_{l}, k_{\perp}\right)=\frac{\xi_{l}-c q_{l} Z_{l}}{\xi_{l}+c q_{l} Z_{l}},
$$

where $Z_{l} \equiv Z\left(\mathrm{i} \xi_{l}\right)$. The Leontovich impedance is applicable even in some cases when $\varepsilon(\omega)$ loses its meaning (e.g. in the frequency region of the anomalous skin effect). If, however, both quantities are in their domain of application, they are related by $Z(\omega)=1 / \sqrt{\varepsilon(\omega)}[20]$. For the impedance functions of the normal and anomalous skin effect, the Ginzburg reflection coefficients at zero frequency satisfy equation (4) as do the Lifshitz ones for ideal metals. In the region of infrared optics it follows that

$$
r_{\|}^{\mathrm{G}}\left(0, k_{\perp}\right)=1, \quad r_{\perp}^{\mathrm{G}}\left(0, k_{\perp}\right)=\frac{\omega_{\mathrm{p}}-c k_{\perp}}{\omega_{\mathrm{p}}+c k_{\perp}} .
$$

The impedance approach to the thermal Casimir force was developed in [21-24]. It predicts small thermal corrections at short separations in agreement with the case of ideal metals and leads to the same high-temperature (large separation) asymptotic results which hold for the plasma model approach or for ideal metals in accordance with the classical limit. Thus, the impedance approach can also be called 'traditional'.

Many computations of the thermal Casimir force have been performed in the framework of the Drude model approach [10-13], plasma model approach [14, 15] and impedance approach [21-24], and many controversial statements in favour and against each approach have appeared in the literature. These arguments were most recently presented in [25, 26]. Before discussing them in more detail, we emphasize that the Drude model and the impedance approaches are qualitatively different only at zero Matsubara frequency (compare equations (6) and (11)). As to the contributions from Matsubara frequencies with $l \geqslant 1$, both approaches find these using tabulated optical data extrapolated to low frequencies by the imaginary part of the Drude dielectric function with practically coinciding results. The plasma model approach applied to all frequencies disregards the internal photoelectric effect (interband transitions) and other processes taken into account in the optical data. Because of this, it is not as accurate as the impedance approach at short separations of about 150-250 $\mathrm{nm}$.

\section{Controversies between the two approaches}

\subsection{Is there a violation of thermodynamics for the Lifshitz formula combined with the Drude model?}

As was proven analytically in [24], the Drude model approach leads to a violation of the third law of thermodynamics (the Nernst heat theorem) in the case of metallic perfect lattices with no defects or impurities. For such lattices the relaxation parameter $\gamma(T) \rightarrow 0$ when $T \rightarrow 0$ in accordance with the Bloch-Grüneisen law (this property is preserved even when the effects of electron-electron collisions are included), and the entropy of a fluctuating field takes the form

$$
S(z, 0)=-\left.\frac{\partial \mathcal{F}(z, T)}{\partial T}\right|_{T=0}=\frac{k_{\mathrm{B}}}{16 \pi z^{2}} \int_{0}^{\infty} y \mathrm{~d} y \ln \left[1-\left(\frac{y-\sqrt{\frac{\omega_{\mathrm{p}}^{2}}{\omega_{c}^{2}}+y^{2}}}{y+\sqrt{\frac{\omega_{\mathrm{p}}^{2}}{\omega_{c}^{2}}+y^{2}}}\right)^{2} \mathrm{e}^{-y}\right]<0
$$

where $\omega_{c}=c /(2 z)$ is the characteristic frequency of the Casimir effect. 
To avoid this conclusion, $[12,13]$ apply the Drude model approach to metallic lattices with defects and impurities possessing some residual relaxation $\gamma(0) \neq 0$ and obtain $S(z, 0)=0$. This, however, does not solve the problem of the thermodynamic inconsistency of the Drude model approach, because perfect lattices have a nondegenerate dynamical state of lowest energy and, thus, according to quantum statistical physics, the entropy at zero temperature must be equal to zero in this case (a property violated by the Drude model approach according to equation (12)). Recently, the authors of [26] recognized that the Drude model approach violates thermodynamics 'for perfect metals of infinite extension' having no relaxation (the so-called 'modified ideal metal' which is obtained from the real metals in the Drude model approach when $\varepsilon \rightarrow \infty$ [26]), but they deny a violation for metals which include relaxation. This denial is based on a misunderstanding. As was already mentioned above, the Drude model approach violates the Nernst heat theorem for metallic perfect lattices with no defects or impurities (see [24] and equation (12)). Such lattices have a nonzero resistance and relaxation at any nonzero temperature, which go to zero only when temperature vanishes. Thus, they are not 'perfect metals' according to [26].

In the light of the above, the conclusion of $[12,26]$ that the Drude model approach is in agreement with thermodynamics cannot be supported. In contrast, the plasma model and the impedance approaches are in complete agreement with thermodynamics [15, 24].

\subsection{Do the 'exact' impedances lead to the same result as the Drude model or as the Leontovich impedance?}

As was mentioned in section 2, the impedance boundary condition (9) is an approximate one. In particular, it works well if the electromagnetic waves penetrating into metal interior propagate almost perpendicular to its surface. (This is in fact the case as long as $|\varepsilon(\omega)| \gg 1$.) Some authors (see, e.g., $[12,27]$ ) introduce the so-called 'exact' impedances

$Z_{\|}\left(\omega, k_{\perp}\right)=Z(\omega)\left(1-\frac{c^{2} k_{\perp}^{2}}{\omega^{2} \varepsilon}\right)^{1 / 2}, \quad Z_{\perp}\left(\omega, k_{\perp}\right)=Z(\omega)\left(1-\frac{c^{2} k_{\perp}^{2}}{\omega^{2} \varepsilon}\right)^{-1 / 2}$,

which depend on both polarization and transverse momentum, and related impedance boundary conditions:

$$
\boldsymbol{E}_{\mathrm{t}}=Z_{\perp}\left(\omega, k_{\perp}\right)\left[\boldsymbol{H}_{\mathrm{t}} \times \boldsymbol{n}\right], \quad Z_{\|}\left(\omega, k_{\perp}\right) \boldsymbol{H}_{\mathrm{t}}=\left[\boldsymbol{n} \times \boldsymbol{E}_{\mathrm{t}}\right]
$$

where $Z(\omega)$ is the Leontovich impedance. Actually, the impedances (13) are not exact. Equations (13) and (14) follow from the Maxwell equations only if $\varepsilon(\omega)$ has definite meaning, so that the spatial dispersion is neglected and the relation $\boldsymbol{D}(\boldsymbol{r}, \omega)=\varepsilon(\omega) \boldsymbol{E}(\boldsymbol{r}, \omega)$ is valid. If, however, the homogeneity of space is violated by the presence of boundaries, and if spatial dispersion is taken into account, equations (13) and (14) are in general inapplicable (see section 5).

In terms of the impedances (13), the reflection coefficients take the form

$$
r_{\|}\left(\xi_{l}, k_{\perp}\right)=\frac{c q_{l}-Z_{l, \|} \xi_{l}}{c q_{l}+Z_{l, \|} \xi_{l}}, \quad r_{\perp}\left(\xi_{l}, k_{\perp}\right)=\frac{\xi_{l}-c q_{l} Z_{l, \perp}}{\xi_{l}+c q_{l} Z_{l, \perp}},
$$

where $Z_{l, \|} \equiv Z_{\|}\left(\mathrm{i} \xi_{l}, k_{\perp}\right)$ and $Z_{l, \perp} \equiv Z_{\perp}\left(\mathrm{i} \xi_{l}, k_{\perp}\right)$. It is easy to check that if one substitutes equation (13) into equation (15), considers $\xi_{l}$ and $k_{\perp}$ as independent variables and takes into account that $Z=1 / \sqrt{\varepsilon}$, the Lifshitz reflection coefficients (2) are recovered. This allowed the authors of $[11,12,26]$ to insist that the results (4) and (11), obtained in $[22,24]$ within the impedance approach (in contrast to equation (6) valid in the Drude model approach), are due to disregarding the transverse momentum dependence by the Leontovich impedance. 
Bearing in mind that equation (6) returns us to the contradiction with thermodynamics, it is necessary to analyse this point more thoroughly. When we are considering real photons incident on a plane boundary of a single semispace, the components of a wave vector are constrained by the mass-shell equation $|\boldsymbol{k}|^{2}=k_{\perp}^{2}+k_{3}^{2}=\omega^{2} / c^{2}\left(k_{3}\right.$ is the wave vector component perpendicular to the boundary). Using this equation, the angle of incidence is expressed by $\sin \vartheta_{0}=k_{\perp} /|\boldsymbol{k}|=c k_{\perp} / \omega$ and the impedances (13) can be identically rewritten in the form

$Z_{\|}\left(\omega, k_{\perp}\right)=Z(\omega)\left[1-\frac{\sin ^{2} \vartheta_{0}}{\varepsilon(\omega)}\right]^{1 / 2}, \quad Z_{\perp}\left(\omega, k_{\perp}\right)=Z(\omega)\left[1-\frac{\sin ^{2} \vartheta_{0}}{\varepsilon(\omega)}\right]^{-1 / 2}$.

The term $\sin ^{2} \vartheta_{0} / \varepsilon(\omega)$ can be neglected for all frequencies which are at least several times smaller than the plasma frequency because in this region $|\varepsilon| \gg 1$. Furthermore, in the limit of zero frequency $\sin ^{2} \vartheta_{0} / \varepsilon \rightarrow 0$ due to $|\varepsilon| \rightarrow \infty$, and as a result for real photons the Leontovich impedance coincides with the impedances $Z_{\|, \perp}$ precisely. The mass-shell equation ensures the fulfilment of Snell's laws. Thus, if the reflection properties for virtual photons are postulated to be the same as for real photons, the Leontovich impedance and boundary condition (9) are recovered.

These considerations can be generalized for the case of two semispaces with the boundary conditions (14). In this case, the Maxwell equations lead to the following dispersion relations for the determination of photon eigen-frequencies $\omega[22]$ :

$$
\begin{aligned}
& \Delta_{\|}\left(\omega, k_{\perp}\right) \equiv \mathrm{e}^{-q z}\left(1-\eta_{\|}^{2}\right)\left(\sinh q z-\frac{2 \mathrm{i} \eta_{\|}}{1-\eta_{\|}^{2}} \cosh q z\right)=0, \\
& \Delta_{\perp}\left(\omega, k_{\perp}\right) \equiv \mathrm{e}^{-q z}\left(1-\kappa_{\perp}^{2}\right)\left(\sinh q z+\frac{2 \mathrm{i} \kappa_{\perp}}{1-\kappa_{\perp}^{2}} \cosh q z\right)=0,
\end{aligned}
$$

where

$$
q^{2}=k_{\perp}^{2}-\omega^{2} / c^{2}, \quad \eta_{\|}=\frac{\omega Z_{\|}\left(\omega, k_{\perp}\right)}{c q}, \quad \kappa_{\perp}=\frac{c q Z_{\perp}\left(\omega, k_{\perp}\right)}{\omega} .
$$

It is easily seen from equations (17) that in the limit $\omega \rightarrow 0$ the transverse momentum must also go to zero, $k_{\perp} \rightarrow 0$, in such a way that $c k_{\perp} \sim \omega$. This means that the quantity $c^{2} k_{\perp}^{2} /\left(\omega^{2} \varepsilon\right)$ in equation (13) goes to zero in the limit of zero frequency, and both impedances coincide precisely with the Leontovich impedance. At all nonzero frequencies there are only negligible differences between the impedances (13) and the Leontovich impedance. Thus, in the case of two semispaces, the dispersion relations (17) play the same role as the mass-shell equation for free photons.

Using the above property of the solutions of equations (17), these equations can be approximately rearranged to the form following from the boundary conditions (9):

$$
\begin{aligned}
& \Delta_{\|}\left(\omega, k_{\perp}\right) \equiv \mathrm{e}^{-q z}\left(1-\eta^{2}\right)\left(\sinh q z-\frac{2 \mathrm{i} \eta}{1-\eta^{2}} \cosh q z\right)=0, \\
& \Delta_{\perp}\left(\omega, k_{\perp}\right) \equiv \mathrm{e}^{-q z}\left(1-\kappa^{2}\right)\left(\sinh q z+\frac{2 \mathrm{i} \kappa}{1-\kappa^{2}} \cosh q z\right)=0,
\end{aligned}
$$

where $\eta=\omega Z /(c q), \kappa=c q Z / \omega$ are now expressed in terms of the Leontovich impedance. Note that the approximation was made only at $\omega \neq 0$, whereas in the limit $\omega \rightarrow 0$, which is of special importance for our problem, equations (17) and (19) remain equivalent.

It is now easy to proceed with the derivation of the Lifshitz formula by summing the free energies of all oscillators whose frequencies are determined by equations (19), applying the argument theorem and calculating the residues at imaginary Matsubara frequencies [22]. 
This derivation results in equation (1) with the reflection coefficients in equation (10). Note that in our derivation the transverse momentum dependence is not 'completely disregarded' (as is claimed in $[11,12,26]$ ). The transverse momentum $k_{\perp}$ enters through the quantity $q$ both in the dispersion equations (19) and in the reflection coefficients (10). We emphasize that the dispersion relations (19) with the Leontovich impedance have both photonic and plasmonic solutions. It is not admissible, however, to preserve $k_{\perp} \neq 0$ in the impedance function $Z_{\perp}\left(\omega, k_{\perp}\right)$, and to consider $\omega \rightarrow 0$ because this violates the dispersion relations (17). The Matsubara frequencies and the transverse momentum become independent only after application of the argument theorem. In view of the above, the conclusion of [12] that the Drude model and impedance approaches lead to the same reflection coefficients at zero frequency is not warranted.

\subsection{How to extrapolate real data to zero frequency?}

As was already discussed in section 2, the tabulated optical data for real materials do not contain the zero-frequency contribution which should be found from some theoretical considerations. There are two main methods proposed in the literature for solving this problem. According to one method (see, e.g., $[12,26]$ ) it is necessary to extrapolate to zero the actual reflection properties of plate materials at very low, quasistatic frequencies. According to another method, the zero-frequency limit should not be understood literally because there are no static-field fluctuations. More likely, it should be understood as a mathematical limit to zero from the region around the characteristic frequency $\omega_{c}$ giving the major contribution to the thermal Casimir force [21-24]. Although in [12] such an extrapolation is considered to be 'ad hoc', it was demonstrated in [23] that the method of [12, 26], applied in the framework of the impedance approach, results in a violation of the Nernst heat theorem.

Recently the method of [21-24] received support when applied to two dielectric semispaces, i.e., to the case which was previously free of any controversies. As was analytically proved in [28] for similar (and in [29] for dissimilar) dielectrics, the Casimir entropy of a fluctuating field goes to zero when temperature vanishes if the semispace materials are described by a frequency-dependent $\varepsilon$ with some finite static values $\varepsilon(0)$. If, however, the dc conductivity of dielectrics is taken into account, this results in a violation of the Nernst heat theorem. When it is recalled that dielectrics really possess some nonzero conductivity at a constant current (although it is many orders of magnitudes smaller than that for metals), it becomes clear that real material properties at very low frequencies are in fact irrelevant to the fluctuating phenomena and must not be included into the model of the dielectric response.

\section{Different approaches to the thermal Casimir force and experiment}

\subsection{Measurement of the Casimir force using a torsion pendulum}

As was mentioned in section 2, the traditional approaches to the thermal Casimir force predict small thermal corrections at short separations in qualitative agreement with the case of ideal metals. The sensitivity of all already performed experiments is not sufficient to measure such corrections, much less to discriminate among different traditional approaches. Some of these experiments are, however, sufficiently sensitive to detect the alternative thermal corrections as predicted by the Drude model approach if they exist.

According to the results of the first modern measurement of the Casimir force between a Au coated plate and large spherical lens [30], at $T=300 \mathrm{~K}$ with $z=1 \mu \mathrm{m}$, a net deviation between the Casimir forces computed for ideal metals and using the Drude model approach 
is $25 \%$. Of this deviation, $19 \%$ is due to the large alternative thermal correction. In [30], the experimental uncertainty at $z=1 \mu \mathrm{m}$ was about $5-10 \%$. The predicted thermal effect was not, however, observed. According to [31], the prediction of the Drude model approach 'deviates significantly from experimental results'. At the same time, according to [32], the experimental data are consistent with the impedance approach.

\subsection{Static experiment using a microelectromechanical torsional oscillator}

In this experiment the Casimir force between a plate of a torsional oscillator and a sphere was measured [33]. The sphere was coated by $\mathrm{Au}$ and the plate by $\mathrm{Cu}$. The force values were measured with an absolute error of $0.3 \mathrm{pN}$ for separations in the range 0.19-1.2 $\mu \mathrm{m}$. Reference [12] claims that there is 'reasonably good agreement' between the Drude model approach and the experimental results of the static experiment at $z=200 \mathrm{~nm}$. In [25] it was demonstrated, however, that this conclusion is based on a misunderstanding of the data. In fact, the experimental data are in contradiction with the Drude model approach, but are consistent with the traditional approaches to the thermal Casimir force. For example, at about $z=200 \mathrm{~nm}$ the mean value of $F^{\text {th }}-F^{\text {exp }}$ (where the theoretical Casimir force $F^{\text {th }}$ is computed using one of the traditional approaches) is practically zero. If the Drude model approach is used, this difference is approximately $2.6 \mathrm{pN}$ (i.e. it is almost nine times larger than the experimental absolute error), thus, upsetting the agreement between experiment and theory.

\subsection{Dynamic experiment using a microelectromechanical torsional oscillator}

In the second, dynamic experiment of [33], the equivalent Casimir pressure between two parallel plates (one coated by $\mathrm{Au}$ and another by $\mathrm{Cu}$ ) was determined dynamically in the separation range from 260 to $1200 \mathrm{~nm}$ with an absolute error of about $0.6 \mathrm{mPa}$. In the comparison between the experimental data and different theoretical approaches, a number of possible corrections to the theoretical Casimir pressures were taken into account. These include those due to surface roughness, the use of the proximity force theorem, the finite sizes of the plates, sample-dependent variations of the tabulated optical data for the complex index of refraction and contribution of nonlocal effects. The differences between the theoretical and experimental Casimir pressures, $\left[P^{\mathrm{th}}(z)-P^{\exp }(z)\right]$, deviate from zero in the range from -0.8 to $0.5 \mathrm{mPa}$ if the traditional theoretical approaches to the thermal Casimir force are used [33]. This demonstrates that the traditional approaches are consistent with the experimental data. If, however, the theoretical Casimir pressures $\tilde{P}^{\text {th }}(z)$ are computed using the Drude model approach, the differences $\left[\tilde{P}^{\text {th }}(z)-P^{\exp }(z)\right]$ deviate significantly from zero within the separation region from 260 to $700 \mathrm{~nm}$. At the shortest separation this deviation reaches $5.5 \mathrm{mPa}$ (i.e. it is more than nine times larger than the experimental absolute error). In [33], the conclusion was drawn that the Drude model approach is ruled out experimentally.

\subsection{Improved dynamic experiment using a microelectromechanical torsional oscillator}

In $[34,35]$ the results of a new, improved, dynamic experiment were reported on the determination of the Casimir pressure between the two plates both coated by $\mathrm{Au}$ in the separation region from 160 to $700 \mathrm{~nm}$. The main improvements were a significant suppression of the surface roughness, a decrease by a factor 1.7 in the error in the measurement of the absolute separations, and the use of rigorous statistical procedures in data processing and in the comparison of experiment and theory. Importantly, the contribution of the surface roughness to the Casimir pressure was reduced to less than $0.65 \%$ even at the shortest separation. This is 


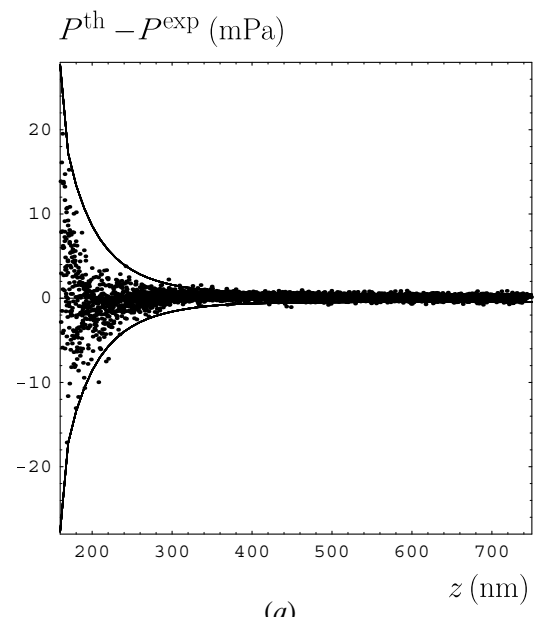

(a)

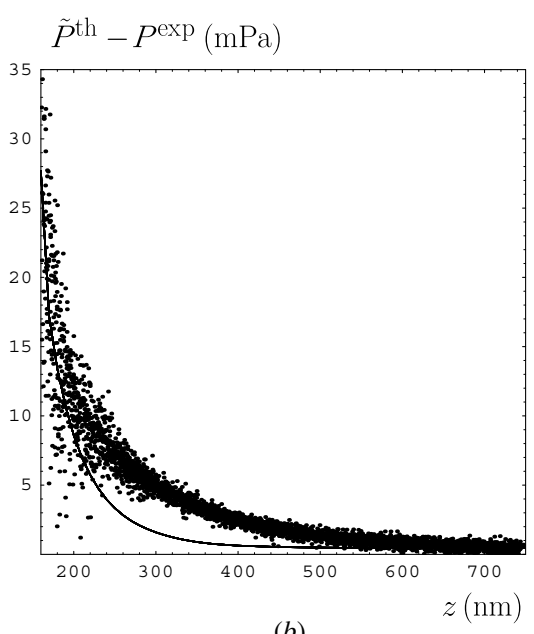

(b)

Figure 1. Differences between the theoretical Casimir pressures calculated using one of the traditional approaches $(a)$ or the Drude model approach $(b)$ and experimental ones versus separation. The $95 \%$ confidence interval lies between the solid lines.

the first experiment where the total relative error of the Casimir pressure measurements found at $95 \%$ confidence varies between 0.54 and $0.59 \%$ in a wide separation region from 170 to $300 \mathrm{~nm}$, and is as small as $2.5 \%$ at $z=500 \mathrm{~nm}$.

In addition to the above-mentioned corrections in the theoretical Casimir pressure, the contribution of patch potentials and errors in the calculated pressures, arising from the uncertainties in the experimental distances [36] were taken into account. Finally, the total theoretical error, which was determined at $95 \%$ confidence, decreases from $1.65 \%$ at $z=160 \mathrm{~nm}$ to $0.9 \%$ at $z=750 \mathrm{~nm}$. Using the total experimental and theoretical errors, determined independently, the absolute error of the pressure differences $\left[P^{\text {th }}(z)-P^{\exp }(z)\right]$ was found at $95 \%$ confidence. It is practically the same in different theoretical approaches. The solid lines in figures $1(a)$ and $(b)$ show the confidence interval versus separation which is determined in terms of this error. In figure $1(a)$ the differences $\left[P^{\text {th }}(z)-P^{\exp }(z)\right]$ for the traditional theories are plotted. In this case more than $95 \%$ of all individual points (not only the mean values of pressure differences at each separation as is demanded by the theorems of mathematical statistics) belong to the confidence interval. This means that our error analysis is in fact too conservative and the errors are significantly overestimated. From figure 1( $a)$ it follows that the traditional approaches to the thermal Casimir force are consistent with data. In figure $1(b)$ the theoretical pressures $\tilde{P}^{\text {th }}(z)$ are computed using the Drude model approach. It is seen that this approach is excluded experimentally within the separation region from 170 to $700 \mathrm{~nm}$ at $95 \%$ confidence. As is shown in [34], in the separation region from 300 to $500 \mathrm{~nm}$ the Drude model approach is excluded by experiment at even higher $99 \%$ confidence.

In the first version of preprint [26], it was claimed that the computations of [34] in the framework of the Drude model approach deviate somewhat from the original computations of [34]. Later it was shown [25] that these deviations were caused by numerical discrepancies in the data used in preprint [26], which was recognized by the authors in the second and third versions of this preprint.

Nevertheless, [26] claims that 'the experimental situation is still too indecisive to draw definite conclusions' on the contradiction of the Drude model approach with experiment. To 
justify this conclusion, [26] cites [36] where the influence of the experimental uncertainty in surface separations on the theoretical values of the Casimir force was discussed. As was mentioned above, the full contribution from this effect is included in the error analysis of [34], and thus cannot be used to cast doubts on the accuracy of the obtained results. Reference [26] also claims that 'the roughness of the surfaces is much larger than the precision stated in the determination of the separation'. This, however, cannot compromise the precision achieved in [34] because the separations are measured between zero levels of surface roughness independently of the value of roughness amplitude. Furthermore, the contribution of roughness in the experiment of [34] is so small that the validity of all the conclusions is preserved even if one were to neglect the roughness in the theoretical analysis. As was demonstrated in [25], no unaccounted fixed systematic error in surface separation is capable of bringing the Drude model approach into agreement with data within the whole range of measurements. One more claim of [26] is that 'accurate determination of a small difference between experimental values at room temperature and purely theoretical values at $T=0$ gives rise to further difficulties'. This claim is evidently misleading because all theoretical values of the Casimir pressure in [34] were computed at $T=300 \mathrm{~K}$, i.e., at the experimental temperature. To summarize, all of the arguments of [12, 26] against the precision and accuracy of the experiment of [34] break down, and the conclusion that there is a contradiction between the Drude model approach and experiment seems unavoidable.

\section{Spatial dispersion and the Casimir force}

Recent papers [37, 38] claim that taking account of the spatial dispersion is crucial for the problem of the thermal Casimir force between real metals. They use the usual (continuity) boundary conditions (3) and the usual Lifshitz formula (1) with the reflection coefficients expressed in terms of the spatially nonlocal dielectric permittivities $\varepsilon_{i}\left(\omega, k_{\perp}\right)$ or impedances $Z_{\|, \perp}\left(\omega, k_{\perp}\right)$ (the latter are presented in terms of nonlocal dielectric permittivities by means of some integral relations). References [37, 38] arrive at the conclusion that the contribution from $r_{\perp}\left(0, k_{\perp}\right)$ is negligibly small, leading to approximately the same results for the thermal Casimir force as in the Drude model approach. In contrast to the Drude model approach, entropy was shown to be equal to zero at $T=0$ even for perfect crystal lattices [38].

As was already demonstrated in section 4, the predictions of the Drude model approach are in contradiction with experiment. There are also serious theoretical objections against the formalisms used in [37, 38]. As follows from Maxwell equations, in the presence of spatial dispersion the boundary conditions for physical fields take the form $[39,40]$

$$
E_{1 t}=E_{2 t}, \quad B_{1 n}=B_{2 n}, \quad D_{2 n}-D_{1 n}=4 \pi \sigma, \quad\left[n \times\left(B_{2}-B_{1}\right]=\frac{4 \pi}{c} \boldsymbol{i},\right.
$$

where

$$
\sigma=\frac{1}{4 \pi} \int_{1}^{2} \operatorname{div}[\boldsymbol{n} \times[\boldsymbol{D} \times \boldsymbol{n}]] \mathrm{d} l, \quad \boldsymbol{i}=\frac{1}{4 \pi} \int_{1}^{2} \frac{\partial \boldsymbol{D}}{\partial t} \mathrm{~d} l
$$

are the induced charge and current surface densities, respectively (assuming that there are no external charges and currents). If spatial dispersion is absent, then $\sigma=0, i=0$, and the usual continuity boundary conditions (3) are recovered [39, 40]. Then the use of boundary conditions (3) in the presence of spatial dispersion is unjustified.

References [37, 38] disregard the fact that spatially nonlocal dielectric permittivities $\varepsilon_{i}\left(\omega, k_{\perp}\right)$ can be rigorously introduced only for an unbounded medium which is uniform in space $[39,40]$. In the theory of the anomalous skin effect, such permittivities are sometimes used in the presence of boundaries (see, e.g., [41]). This is an approximation applicable to the 
investigation of some bulk effects, when physical phenomena caused by a layer adjacent to the boundary surface are neglected. (There is another approach to the theory of the anomalous skin effect [42] which only deals with the Leontovich impedance.) In any case, it is unlikely that the approximate phenomenological approach using nonlocal $\varepsilon_{i}\left(\omega, k_{\perp}\right)$ in the presence of boundaries would be applicable in the theory of Casimir force between metal surfaces, where the boundary effects are of prime importance.

It has also been known [43] that with inclusion of spatial dispersion the free energy of a fluctuating field $\tilde{\mathcal{F}}(z, T)$ takes a more general form than is given by the Lifshitz formula, i.e.,

$$
\tilde{\mathcal{F}}(z, T)=\mathcal{F}(z, T)+\Delta \mathcal{F}(z, T),
$$

where $\mathcal{F}(z, T)$ is the Lifshitz contribution presented in equation (1), and the general expression for $\Delta \mathcal{F}(z, T)$ in terms of the thermal Green's functions of the electromagnetic field can be found in [43]. The use of the Lifshitz free energy $\mathcal{F}(z, T)$ for systems with spatial dispersion (as was done, e.g., in [44] where $\varepsilon_{i}\left(\omega, k_{\perp}\right)$ of the hydrodynamic model were substituted into $\mathcal{F}$ ) is not correct [43]. As was emphasized in [43], 'for most of condensed matter bodies this is inadmissible'. It seems, however, that $[37,38]$ repeat this misuse of the Lifshitz formula. At the same time, it is amply evident that at $T=300 \mathrm{~K}$ in the region of infrared optics (i.e. at the experimental separations) or in the region of the normal skin effect $(z>4-5 \mu \mathrm{m})$ spatial dispersion does not play any role and can be neglected.

\section{Conclusions}

From the above, the following conclusions can be formulated:

- The thermal Casimir force between real metals presents serious problems, and there is presently no fundamental theory which describes it;

- The Drude model approach to the thermal Casimir force violates the Nernst heat theorem in the case of metallic perfect crystal lattices with no impurities and is excluded by experiment;

- The thermal effect in the Casimir force has not yet been measured. The traditional approaches to its description based on the plasma model and on the Leontovich impedance are consistent with experiment. Computations using the Lifshitz formula at $T=0$ and tabulated optical data are also consistent with experiment;

- The measurement of the small thermal effect, as predicted by the traditional approaches, would be of great interest and could help to discriminate between them. In this regard the proposed experiments $[32,45,46]$ are very promising;

- The desired fundamental theory of the thermal Casimir force between real materials should be presumably based on a more sophisticated quantization procedure incorporating spatial dispersion and inhomogeneity of space, and may go beyond the scope of the Lifshitz theory.

\section{Acknowledgments}

VBB and CR were partially supported by CNPq. EF was supported by DOE Grant no DEAC02-76ER071428. The work of GLK and VMM was supported by DFG Grant no 436 RUS $113 / 789 / 0-1$.

\section{References}

[1] Lifshitz E M and Pitaevskii L P 1980 Statistical Physics Part II (Oxford: Pergamon)

[2] Casimir H B G 1948 Proc. K. Ned. Akad.Wet. 51793 
[3] Milonni P W 1994 The Quantum Vacuum (San Diego: Academic)

[4] Mostepanenko V M and Trunov N N 1997 The Casimir Effect and its Applications (Oxford: Clarendon)

[5] Milton K A 2001 The Casimir Effect (Singapore: World Scientific)

[6] Bordag M, Mohideen U and Mostepanenko V M 2001 Phys. Rep. 3531

[7] Lifshitz E M 1956 Sov. Phys._JETP 273

[8] Brown L S and Maclay G J 1969 Phys. Rev. 1841272

[9] Boström M and Sernelius B E 2000 Phys. Rev. Lett. 844757

[10] Høye J S, Brevik I, Aarseth J B and Milton K A 2003 Phys. Rev. E 67056116

[11] Milton K A 2004 J. Phys. A: Math. Gen. 37 R209

[12] Brevik I, Aarseth J B, Høye J S and Milton K A 2005 Phys. Rev. E 71056101

[13] Boström M and Sernelius B E 2004 Physica A 33953

[14] Genet C, Lambrecht A and Reynaud S 2000 Phys. Rev. A 62012110

[15] Bordag M, Geyer B, Klimchitskaya G L and Mostepanenko V M 2000 Phys. Rev. Lett. 85503

[16] Jancovici B and Šamaj L 2005 Europhys. Lett. 7235

[17] Buenzli P R and Martin P A 2005 Europhys. Lett. 7242

[18] Feinberg J, Mann A and Revzen M 2001 Ann. Phys. NY 288103

[19] Kats E I 1977 Sov. Phys._JETP 46109

[20] Landau L D, Lifshitz E M and Pitaevskii L P 1984 Electrodynamics of Continuous Media (Oxford: Pergamon)

[21] Bezerra V B, Klimchitskaya G L and Romero C 2002 Phys. Rev. A 65012111

[22] Geyer B, Klimchitskaya G L and Mostepanenko V M 2003 Phys. Rev. A 67062102

[23] Geyer B, Klimchitskaya G L and Mostepanenko V M 2004 Phys. Rev. A 70016102

[24] Bezerra V B, Klimchitskaya G L, Mostepanenko V M and Romero C 2004 Phys. Rev. A 69022119

[25] Bezerra V B, Decca R S, Fischbach E, Geyer B, Klimchitskaya G L, Krause D E, López D, Mostepanenko V M and Romero C 2005 Preprint quant-ph/0503134

Bezerra V B, Decca R S, Fischbach E, Geyer B, Klimchitskaya G L, Krause D E, López D, Mostepanenko V M and Romero C Phys. Rev. E at press

[26] Høye J S, Brevik I, Aarseth J B and Milton K A 2005 Preprint quant-ph/0506025

[27] Esquivel R, Villarreal C and Mochán W L 2003 Phys. Rev. A 68052103

[28] Klimchitskaya G L, Geyer B and Mostepanenko V M 2006 J. Phys. A: Math. Gen. 396495

[29] Geyer B, Klimchitskaya G L and Mostepanenko V M 2005 Phys. Rev. D 72085009

[30] Lamoreaux S K 1997 Phys. Rev. Lett. 785

[31] Torgerson J R and Lamoreaux S K 2004 Phys. Rev. E 70047102

[32] Lamoreaux S K and Buttler W T 2005 Phys. Rev. E 71036109

[33] Decca R S, Fischbach E, Klimchitskaya G L, Krause D E, López D and Mostepanenko V M 2003 Phys. Rev. D 68116003

[34] Decca R S, López D, Fischbach E, Klimchitskaya G L, Krause D E and Mostepanenko V M 2005 Ann. Phys. NY 31837

[35] Klimchitskaya G L, Decca R S, Fischbach E, Krause D E, López D and Mostepanenko V M 2005 Int. J. Mod. Phys. A 202205

[36] Iannuzzi D, Gelfand I, Lizanti M and Capasso F 2004 Quantum Field Theory Under the Influence of External Conditions (Princeton: Rinton) p 11

[37] Sernelius B E 2005 Phys. Rev. B 71235114

[38] Svetovoy V B and Esquivel R 2005 Phys. Rev. E 72036113

[39] Agranovich V M and Ginzburg V L 1984 Crystal Optics with Spatial Dispersion, and Excitons (Berlin: Springer)

[40] Ginzburg V L 1985 Physics and Astrophysics (Oxford: Pergamon)

[41] Kliewer K L and Fuchs R 1968 Phys. Rev. 172607

[42] Kaganova I M and Kaganov M I 2001 Phys. Rev. B 63054202

[43] Barash Yu S and Ginzburg V L 1975 Sov. Phys. Usp. 18305

[44] Kleinman G G and Landman U 1974 Phys. Rev. Lett. 33524

[45] Chen F, Klimchitskaya G L, Mohideen U and Mostepanenko V M 2003 Phys. Rev. Lett. 90160404

[46] Brown-Hayes M, Dalvit D A R, Mazzitelli F D, Kim W J and Onofrio R 2005 Phys. Rev. A 72052102 